\title{
Review of Exchange Processes on Ganymede in View of Its Planetary Protection Categorization
}

\author{
O. Grasset, E.J. Bunce, ${ }^{2}$ A. Coustenis, ${ }^{3}$ M.K. Dougherty, ${ }^{4}$ C. Erd, ${ }^{5}$ \\ H. Hussmann, ${ }^{6}$ R. Jaumann, ${ }^{6}$ and O. Prieto-Ballesteros ${ }^{7}$
}

\begin{abstract}
In this paper, we provide a detailed review of Ganymede's characteristics that are germane to any consideration of its planetary protection requirements. Ganymede is the largest moon in our solar system and is the subject of one of the main science objectives of the JUICE mission to the jovian system. We explore the probability of the occurrence of potentially habitable zones within Ganymede at present, including those both within the deep liquid ocean and those in shallow liquid reservoirs. We consider the possible exchange processes between the surface and any putative habitats to set some constraints on the planetary protection approach for this moon. As a conclusion, the "remote" versus "significant" chance of contamination will be discussed, according to our current understanding of this giant icy moon. Based on the different estimates we investigate here, it appears extremely unlikely that material would be exchanged downward through the upper icy layer of Ganymede and, thus, bring material into the ocean over timescales consistent with the survival of microorganisms. Key Words: Planetary science-Planetary protection-Ice. Astrobiology 13, 991-1004.
\end{abstract}

\section{Introduction-Habitability of the Icy Moons}

\subsection{Generalities about the habitability of icy moons}

$\mathbf{T}$ HE ABILITY of a planetary environment to support and sustain life-forms, that is, for it to be a "potentially habitable environment," is based on a combination of factors that are considered to be essential for life to originate, evolve, and be maintained. Some important factors are the orbital properties of the planetary body, its stability, its bulk composition, the existence of an atmosphere and a surface, and proper chemical ingredients (organic chemistry developing in the atmosphere or on the surface or in underground liquid water reservoirs).

The "classical" criteria for potential habitability can be summarized as the presence of liquid water, energy sources to sustain metabolism, and "nutrients" over a period of time long enough to allow the development of life. The concept of a habitable zone around each star defines where water can be stable at the surface as a result of the equilibrium temperature of the planet in the star's radiation field, but recent observations in the outer Solar System have demonstrated that the surface is not the only place where we should search for liquid water.

Indeed, habitable conditions that satisfy the requirements listed above can be found elsewhere besides the surfaces of Earth-like planets. For instance, a subsurface ocean within the satellite of a gas giant may become habitable under certain conditions. Icy crusts may cover liquid oceans, move and fracture by way of tectonics, and expel the internal material and energy through an interconnected system. With the discovery of planets beyond the Solar System and the current or planned extensive search for life in exotic potential habitats such as Mars, Europa, Titan, and Enceladus, habitability in general has attained a much broader definition.

The outer planet satellites are indeed a rich and diverse set of planetary bodies, with great relevance to astrobiological studies, potentially satisfying a number or all of the prerequisites for habitability. Some of them show evidence for organic chemistry in their atmospheres, surfaces, or interiors. Many of the satellites, including the smallest, thus contain organic material. The Cassini-Huygens mission to the saturnian system arrived in July 2004 and deposited a lander

\footnotetext{
${ }^{1}$ Planetology and Geodynamics, University of Nantes, CNRS, France.

${ }^{2}$ Department of Physics and Astronomy, University of Leicester, Leicester, United Kingdom.

${ }^{3}$ LESIA-Observatoire de Paris, CNRS, UPMC Université Paris 06, Université Paris Diderot, France.

${ }^{4}$ Imperial College, London, United Kingdom.

${ }^{5}$ ESA/ESTEC, Noordwijk, the Netherlands.

${ }^{6}$ DLR, Institute of Planetary Research, Berlin, Germany.

${ }^{7}$ Centro de Astrobiología-INTA-CSIC, Madrid, Spain.
} 
on Titan's surface in January 2005. The Cassini orbiter has since been continuously monitoring the saturnian system, an important aspect of which is the environments of the saturnian satellites, including Enceladus, a moon ejecting large quantities of water vapor into space (e.g., Dougherty et al., 2006; Porco et al., 2006). Titan, the largest kronian moon, is organic-rich in the atmosphere through the combination of nitrogen and methane (Coustenis and Taylor, 2008; Vuitton et al., 2009; Waite et al., 2010), with precipitation and lakes of ethane and methane forming on the surface (Stofan et al., 2007, Turtle et al., 2011). Organic components were also measured in Enceladus' plumes (Waite et al., 2006). Enceladus is not the only one of the outer planets' icy moons to show evidence for past and possibly present geological activity. For instance, Neptune's large icy moon Triton is probably a captured Kuiper Belt object and shows plume activity to date. Its surface age is only $\sim 100$ million years, implying that Triton may still be active today (Stern and McKinnon, 2000). In addition, several of the larger satellites are believed to hide global-scale oceans within, as may Enceladus, one of the smaller examples. Both Titan and Enceladus have been described as potential candidates for harboring deep oceans based upon gravity measurements and models of tides (Iess et al., 2012). During the earlier Galileo mission (which arrived at Jupiter in 1995 and was the first spacecraft to orbit Jupiter and closely investigate Europa), additional strong evidence for the presence of an internal ocean (Kivelson et al., 2000) was discovered. Since then, further evidence has accumulated for similar subsurface liquid water oceans to exist not just on Europa but also on the two other icy Galilean satellites: Ganymede and Callisto (Zimmer et al., 2000; McCord et al., 2001). After 8 years in the jovian system, the Galileo mission was terminated by sending the orbiter into Jupiter's atmosphere at a speed of over $48 \mathrm{~km} / \mathrm{s}$ to avoid any chance of its contaminating local moons with terrestrial bacteria. All these outer planetary missions (Voyager, Galileo, and Cassini) have demonstrated that the potentially habitable zone in our solar system is more extended than was previously thought and that the outer planets' satellites can be very well categorized as habitable worlds. The issue of determining the policy for applying adequate planetary protection measures for one of these potentially habitable worlds, Ganymede, is the scope of this paper.

Four types of habitats in the Universe were proposed by Lammer et al. (2009). The first type represents bodies on which stellar and physical conditions allow Earth-analog planets to evolve so that complex multicellular life-forms may originate. A second class includes bodies that may have been habitable in the past, and on which simple life-forms may have emerged, but due to stellar and/or physical conditions the planets evolved toward Venus- or Mars-type worlds where complex life-forms did not develop. These first two types constitute "surface habitats." With the recent emerging evidence for possible internal oceans within the icy moons and detection of life-forms in Earth's mid-ocean ridges in 1977 (Lonsdale, 1977), two new additional types of habitats known as "deep habitats" must also be considered both in the Solar System and beyond. First, there are planetary bodies where subsurface water oceans probably exist, such as on Europa, which interact directly with a silicate-rich core (Fig. 1, right). Second, there are habitats with liquid water layers trapped in between an icy crust on top and a thick icy layer on bottom (Fig. 1, left). The latter is due to the pressure effect on water, which cannot be stable in its liquid form above a few kilobar (Bridgman, 1912, 1937; Choukroun and Grasset, 2010). As soon as the water layer is thick enough (Ganymede, Titan, Callisto, exoplanetary waterworlds), there is no possibility to have a liquid layer in contact with the silicates.
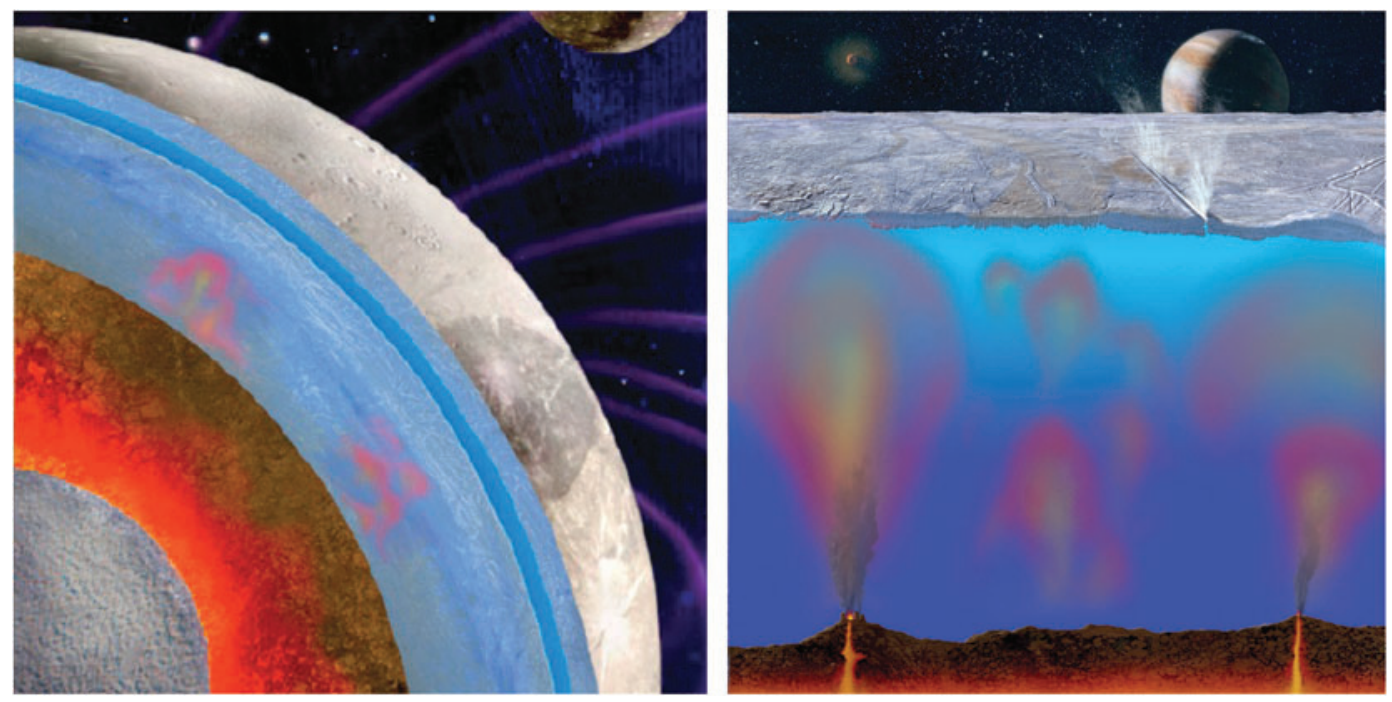

FIG. 1. The two types of possible deep habitats. Left: Ganymede-like habitats where the liquid layer is trapped between an upper icy crust and a thick high-pressure icy mantle. Oceans of these worlds may be habitable only if vigorous exchange processes occur in the thick icy mantle such that they provide complex chemistry and energy sources to the liquid reservoir (credits: Mike Carroll). Right: Europa-like habitats. These worlds could be formed of a hydrosphere no thicker than about $100 \mathrm{~km}$ such that liquid can be in contact with silicates at the bottom of the ocean. At the bottom, conditions are very similar to those encountered in mid-ocean ridges on Earth, where intense biological activity has been discovered (adapted from Clark et al., 2011) (credits: Mike Carroll). Color images available online at www.liebertonline.com/ast 
The role of organic material is vital to the potential habitability of the moons. The fundamental biochemistry required for the life that we know is based on carbon compounds: amino acids, nucleotide bases, sugars, alcohols, and fatty acids. C, H, O, N, P, S are the chemical building blocks of life, but other elements such as $\mathrm{Na}, \mathrm{Mg}, \mathrm{K}, \mathrm{Ca}, \mathrm{Fe}$ are also major components (Wackett et al., 2004). These cations may come from thermal alteration of minerals such as silicates or sulfides, which may be used as a chemical source of energy. The physicochemistry and composition of the deep environments are still unknown, and it is not obvious that they are always the same. Nevertheless, it is commonly agreed that liquid in deep reservoirs is not solely constituted of pure $\mathrm{H}_{2} \mathrm{O}$. It is likely that salty materials such as sulfates or chlorides are trapped within the moons (Kargel et al., 2000; Kivelson et al., 2000; McCord et al., 2001). Many other compounds have been observed on the surfaces or in the atmosphere/plumes of several such moons and are probably present in their interior solutions. $\mathrm{CO}_{2}$ was reported on Europa, Ganymede, Callisto, and Enceladus; $\mathrm{CH}_{4}$ was reported on Enceladus and Titan. Ammonia has been invoked to be in the oceans of Ganymede, Enceladus, or Titan and could also be the source of nitrogen in Enceladus' plume material (Waite et al., 2009; Sohl et al., 2010).

All the above demonstrates that indeed serious consideration of planetary protection measures should be given to the icy satellites of the outer Solar System and in particular to those that will be explored in the future by space missions, such as the JUpiter ICy moons Explorer (JUICE), which has recently been selected for further study toward implementation by the European Space Agency.

\subsection{Planetary protection considerations}

Our current understanding of the deep habitats gained from previous missions has raised the question of the necessary measures regarding planetary protection procedures for any future mission. Many of the science questions relate to the prospects for life and potential for habitability in the Solar System-either due to the potential to find life within the outer planet systems themselves or by improving our understanding of the potential contribution to the origin of life on Earth by Solar System material that may have originated (and may still be found) beyond Earth. As a consequence, some of the future mission opportunities and their potential encounters with habitable zones raise serious questions about biological or organic forward contamination that may be caused by these missions. For these reasons, the COSPAR Panel on Planetary Protection recommended in 2002 the adoption of a simplified version of the Coleman-Sagan equation (Sagan and Coleman, 1966), which is used for establishing the probability of contaminating Europa by Earth microorganisms (Task Group on the Forward Contamination of Europa, 2000). It has also been suggested that the following factors must be quantified prior to any mission toward the outer system:

- Bioburden at launch;

- Cruise survival for contaminating organisms;

- Organism survival in the radiation environment adjacent to the icy world;

- Probability of landing;

- The mechanisms and timescales of transport of organisms to the subsurface liquid water;
- Organism survival and proliferation before, during, and after subsurface transfer.

More recently, a report from the US National Academy of Sciences (Committee on Planetary Protection Standards for Icy Bodies in the Outer Solar System, 2012) proposes a different approach. It is suggested that the Coleman-Sagan formula, even in its simplified form, should not be used with regard to the outer Solar System since many parameters cannot be estimated with adequate accuracy or confidence. One other key issue is that some factors of the formula are not independent. For example, survival of organisms during the different phases (cruise, within the radiation environment of an icy moon, during transport to the subsurface...) is linked in the sense that it all relates to the capacity for an organism to resist the harsh conditions in the surrounding space environment, especially charged-particle radiation, varying pressures, and low temperatures. Hence, another approach could be proposed in which a binary decision framework is used. The decision framework would consider the potential habitability of the planetary object, including environmental conditions necessary for the propagation of terrestrial life; the probability of transport to a subsurface and possibly to the habitable environment; and the ability for terrestrial organisms to survive nominal bioload reduction treatments and adapt to nonterrestrial environments. In the Assessment of Planetary Protection Requirements for Spacecraft Missions to Icy Solar System Bodies (Committee on Planetary Protection Standards for Icy Bodies in the Outer Solar System, 2012), it is suggested that adequate planetary protection should not rely on the multiplication of probabilities (Coleman-Sagan formula) but should employ a series of binary decisions that consider one factor at a time. That would allow us to determine the appropriate level of planetary protection measures to use.

A new categorization of moons and small bodies in the outer Solar System has been recently proposed by Rummel et al. (2009). In this report, the different bodies are classified by the following basis:

Category I: Not of direct interest for understanding the process of chemical evolution.

Category II: Of significant interest relative to the process of chemical evolution and the origin of life, but only a remote chance that contamination by a spacecraft could compromise future investigations. "Remote" means either the absence of niches (places where terrestrial microorganisms could proliferate) or a very low likelihood of transfer to those places.

Category III/IV: Of significant interest relative to the process of chemical evolution and the origin of life and for which scientific opinion provides a significant chance that contamination by spacecraft could compromise future investigations. "Significant" implies that the presence of niches is highly possible and that there is some likelihood of transfer to those places by a plausible mechanism.

The proposed categorization is summarized in Table 1 . One might consider that planetary bodies in Categories I and II are those for which there is a general consensus about the absence of present habitats, or the very low probability for any viable organism to contaminate the putative habitat (binary decision). Thus, the appropriate level of planetary protection measures is well known (no consideration for Category I objects, minimal standards for Category II 
Table 1. Categorization of Moons and Small Bodies of the Outer Solar System (from Rummel ET al., 2009)

\begin{tabular}{|c|c|c|c|}
\hline Group/Body of interest & $\begin{array}{l}\text { Previous } \\
\text { categorization }\end{array}$ & $\begin{array}{c}\text { COSPAR } \\
\text { categorization } \\
\text { (2009) }\end{array}$ & Justification \\
\hline S-Type asteroids & $\mathrm{I}$ & $\mathrm{I}$ & Not of interest to OCE and OOL \\
\hline P-, D-, C-Type asteroids & II & II & $\begin{array}{l}\text { Of interest to OCE and OOL but remote } \\
\text { contamination concern }\end{array}$ \\
\hline Comets & II & II & $\begin{array}{l}\text { Of interest to OCE and OOL but remote } \\
\text { contamination concern }\end{array}$ \\
\hline Io & TBD & I & Not of interest to OCE and OOL \\
\hline Europa & $\mathrm{III} / \mathrm{IV}$ & III/IV & $\begin{array}{l}\text { Of interest to OCE and OOL, significant } \\
\text { contamination concern }\end{array}$ \\
\hline Ganymede & TBD & $\mathrm{II}^{*}$ & $\begin{array}{l}\mathrm{TBC} / \mathrm{Of} \text { interest to } \mathrm{OCE} \text { and } \mathrm{OOL} \text {, nonzero } \\
\text { contamination concern }\end{array}$ \\
\hline Callisto & TBD & II & $\begin{array}{l}\text { Of interest to OCE and OOL but remote } \\
\text { contamination concern }\end{array}$ \\
\hline Other jovian satellites & TBD & II & $\begin{array}{l}\text { Of interest to OCE and OOL but remote } \\
\text { contamination concern }\end{array}$ \\
\hline Titan & TBD & $\mathrm{II}^{*}$ & $\begin{array}{l}\text { TBC/Of interest to OCE and OOL, nonzero } \\
\text { contamination concern }\end{array}$ \\
\hline Enceladus & TBD & III/IV & $\begin{array}{l}\text { Of interest to OCE and OOL, significant } \\
\text { contamination concern }\end{array}$ \\
\hline Other saturnian satellites & TBD & II & $\begin{array}{l}\text { Of interest to OCE and OOL but remote } \\
\text { contamination concern }\end{array}$ \\
\hline Uranian satellites & TBD & II & $\begin{array}{l}\text { Of interest to OCE and OOL but remote } \\
\text { contamination concern }\end{array}$ \\
\hline Triton & TBD & $\mathrm{II}^{*}$ & $\begin{array}{l}\text { TBC/Of interest to OCE and OOL, nonzero } \\
\text { contamination concern }\end{array}$ \\
\hline Other neptunian satellites & TBD & II & $\begin{array}{l}\text { Of interest to OCE and OOL but remote } \\
\text { contamination concern }\end{array}$ \\
\hline Pluto-Charon & II & $\mathrm{II}^{*}$ & $\begin{array}{l}\text { TBC/Of interest to OCE and OOL, nonzero } \\
\text { contamination concern }\end{array}$ \\
\hline Other KBOs: $>1 / 2$ size of Pluto & II & $\mathrm{II}^{*}$ & $\begin{array}{l}\text { TBC/Of interest to OCE and OOL, nonzero } \\
\text { contamination concern }\end{array}$ \\
\hline Other KBOs: $\leq 1 / 2$ size of Pluto & II & II & $\begin{array}{l}\text { Of interest to OCE and OOL but remote } \\
\text { contamination concern }\end{array}$ \\
\hline
\end{tabular}

All missions except Category I need to avoid spacecraft-induced habitats.

*Further data required.

OCE, organic chemical evolution; OOL, origin of life; TBD, to be determined; TBC, to be confirmed; KBOs, Kuiper Belt objects. See text for details.

objects). Categories III/IV indicate that no binary decision can be made regarding the likelihood of contamination if one uses only the two factors, which are the putative presence of habitats and the possible exchange processes to these potential habitats (Europa and Enceladus). Thus, further studies in which one of the two approaches described above is used are needed to determine the appropriate level of planetary protection measures. Finally, a Category $\mathrm{II}^{*}$ has been proposed for three bodies: Ganymede, Titan, and Triton. These three moons require further studies to assess the possibility, the timescale, and the mechanisms of transport of any organism from the surface to the liquid layer.

In this paper, the specific case of Ganymede, the main target of the JUICE mission currently selected by ESA as the first Large mission in the Cosmic Vision 2015-2025 program, is considered. In the next section, a detailed review of the characteristics and the probability of occurrence of habitable zones within Ganymede is proposed, including in the deep liquid ocean and in the shallow aqueous reservoirs if they exist. The third part is focused on the possible exchange processes between the surface and the putative habitats to put some additional constraints on the planetary protection categorization for this moon. As a conclusion, the "remote" versus "significant" chance of contamination will be discussed, based on our current understanding of this giant icy moon.

\section{Habitable Zones in Ganymede}

Ganymede is the largest satellite in the Solar System ( $2631 \mathrm{~km}$ in radius). In the jovian system, it holds a key position because it features old, densely cratered terrain, similar to most of Callisto's surface, but also widespread tectonically resurfaced regions that resemble a large part of Europa's surface. Ganymede displays a wide range of surface ages, which reveal a geological record of several billions of years, and a great variety in geological and geomorphic units. These features are the surface signature of internal heat release during Ganymede's evolution. Ganymede is also the only satellite and-with Mercury and Earth-one of only three solid bodies in the Solar System that generate a magnetic dipole field at the present time. A description of our current knowledge of Ganymede's characteristics can be found in the work of Grasset et al. (2013) and references therein. As indicated by its small moment of inertia factor of 0.3115 (Schubert et al., 2004), Ganymede is a highly differentiated body as the one illustrated in Fig. 1 (left). Interior 

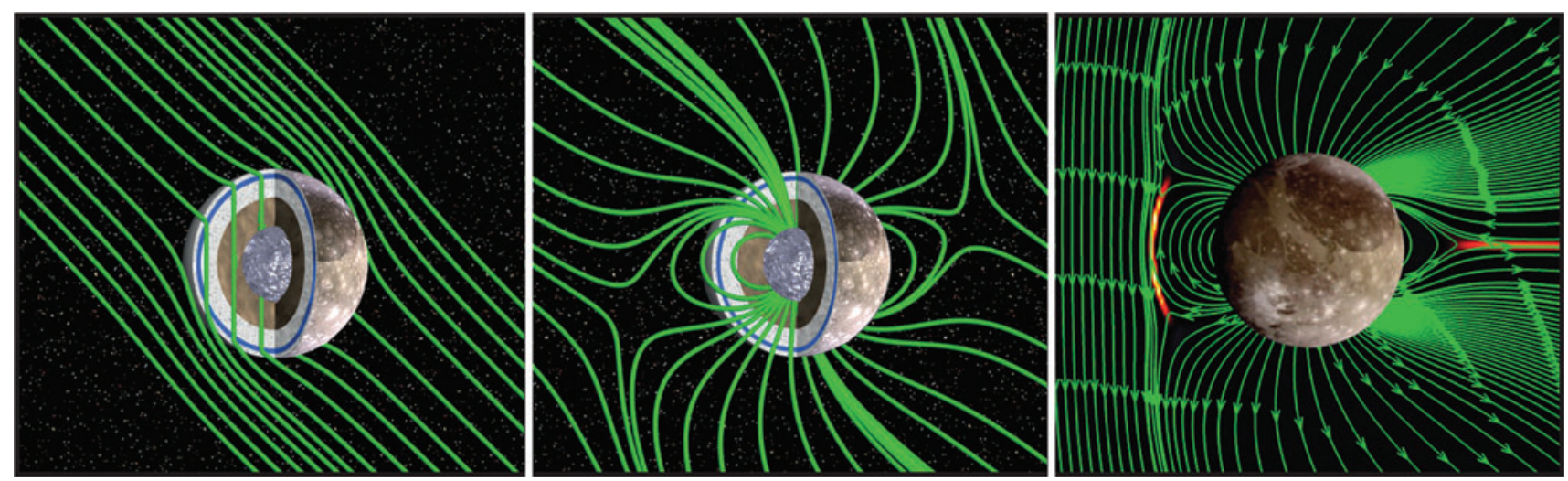

FIG. 2. Ganymede's induced field (left), internally generated magnetic field (middle), and resulting miniature magnetosphere (right). The induced magnetic field is most certainly generated by electrical currents in a liquid ocean trapped between the icy crust and the high-pressure icy layer. [Credits: X. Jia (University of Michigan) and M. Kivelson (UCLA).] Color images available online at www.liebertonline.com/ast

structure models consistent with the gravity field, bulk density, and magnetic constraints include (i) an iron-rich core (at least part of which being liquid to generate the intrinsic magnetic dipole field), (ii) a silicate shell, (iii) a hydrosphere that may be at least $500 \mathrm{~km}$ thick (about $50 \% \mathrm{wt}$ ) and a very tenuous atmosphere (Anderson et al., 1996; Kivelson et al., 2002; Sohl et al., 2002; Spohn and Schubert, 2003).

\subsection{Habitability of the deep ocean}

Voyager and Galileo data indicate that Ganymede possesses some of the prerequisites to be considered potentially habitable. Electrical currents in oceans that contain dissolved salts-and hence provide excellent electrical conductivitygenerate secondary magnetic fields in response to the external rotating jovian magnetic field. Such an induced field was detected by the Galileo spacecraft at Ganymede and provides strong evidence for a present-day subsurface liquid water ocean (Kivelson et al., 2000, 2002). Kivelson et al. (2002) suggested that the depth of the ocean below the surface is close to $150 \mathrm{~km}$. The illustration in Fig. 1 (left) is consistent with this estimate. However, the available data do not allow the depth of the ocean to be accurately constrained. This is because the induced field has not been fully characterized from the Galileo data due to its complex interaction with Ganymede's intrinsic field, Jupiter's magnetosphere, and the plasma environment (Kivelson et al., 2002; Kivelson, 2004; see Fig. 2).

In the large icy satellites of Jupiter, deep aqueous reservoirs likely exist almost independently of the input of stellar energy. Here, tidal dissipation and radiogenic energy keep the water liquid (e.g., Spohn and Schubert, 2003; Hussmann et al., 2006; Schubert et al., 2010). Considering the pressure range encountered within the icy moons, four different scenarios can be envisaged, depending on the varying thicknesses of the water-ice layers and the liquid ocean with respect to the silicate floor (Fig. 3). Case 2 in Fig. 3 is the most probable one for Ganymede in its present state, while case 3 is more probable for Europa and smaller icy moons elsewhere in the Solar System if they host liquid reservoirs (Sohl et al., 2010). Indeed, internal pressures are sufficient to allow for the formation of high-pressure ice phases below the ocean. The hydrosphere is split into a high-pressure ice layer that consists of various water-rich high-pressure ices denser than liquid water, the subsurface water ocean, and an ice-I layer that forms the outer crust of the satellite.

The thickness of the deep ocean is unknown, as discussed above. The depth could be as much as $500 \mathrm{~km}$ if the icy crust were less than $10 \mathrm{~km}$ thick (Spohn and Schubert, 2003). A more reasonable value, assuming an upper crust that is about $100 \mathrm{~km}$ thick (Kivelson et al., 2002), could be in between 100 and $300 \mathrm{~km}$. In this depth of ocean, the pressure would range from $100 \mathrm{MPa}$ at the top to $300-1000 \mathrm{MPa}$ at the bottom. Recent discoveries have shown that life may well adapt to such conditions. For example, pressure may affect the reactivity of simple organic molecules by inducing polymerization (e.g., Hazen et al., 2002; Citroni et al., 2005), promoting catalytic reactions, and hence playing a role in the stability and conformation of biomolecules (Gruner, 1989; Michels and Clark, 1997; Brooks et al., 2011); or pressure may change the equilibrium constants of the solutions associated with life (e.g., Hamman, 1982). Some extreme barophilic microorganisms can survive and be active at pressures up to $1 \mathrm{GPa}$, such as endospores of proteolytic type B Clostridium botulinum TMW 2.357 and Bacillus amyloliquefaciens TMW 2.479 (e.g., San Martín et al., 2002; Margosch et al., 2006).

Chemical and energy exchange between the rocky layer and the ocean, which are so important for habitability, cannot be ruled out but imply efficient transport processes through the thick high-pressure icy layer. Such processes are indeed possible (Sohl et al., 2010) but not as clear-cut as the exchange envisaged for Europa that probably prevailed until recent times. The resurfacing by faulting presumably occurred during periods of high tidal flexing activity and heating. During those past periods of extreme heating, rise of deep melt plumes into Ganymede's ocean may also have been produced (Barr and Stillman, 2011).

As a conclusion, our current knowledge of Ganymede's ocean indicates that it may possess all the prerequisites to be habitable. In the next section, we will study whether exchange from the surface to this putative habitat can be envisaged, and on what timescale.

\subsection{Habitability of shallow reservoirs}

Recent or present geological activity, involving water rising from the shallow subsurface (mainly by cryomagmatism), 


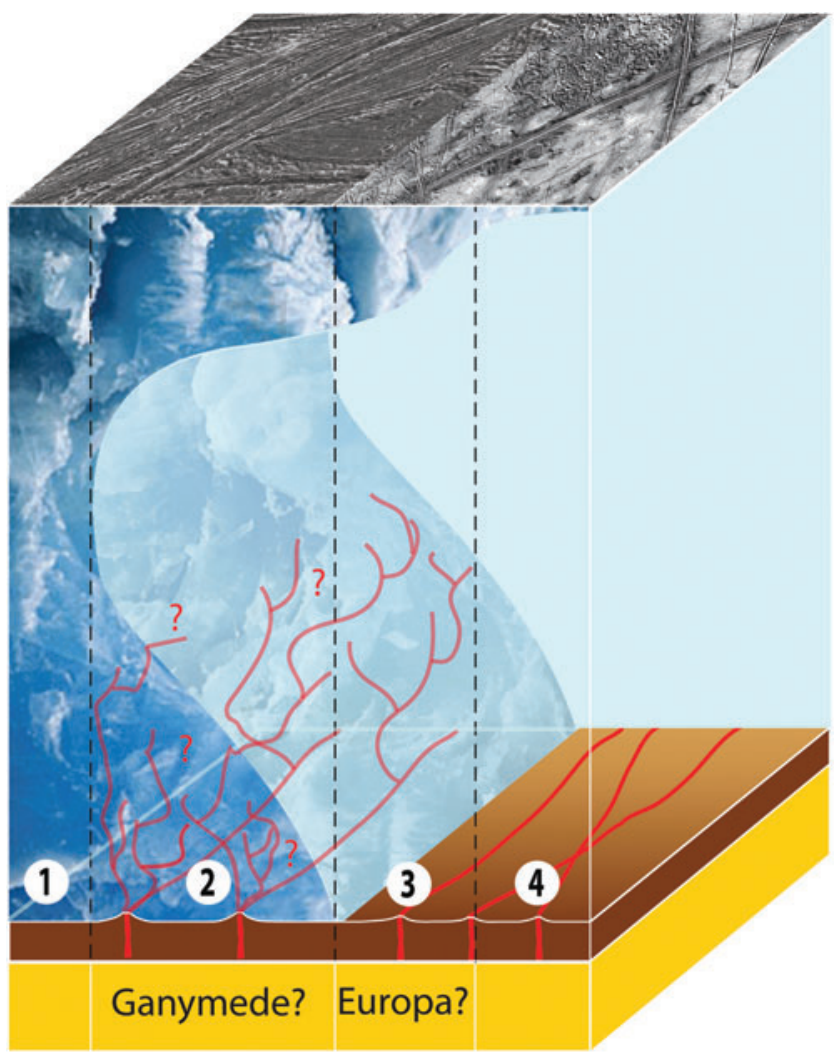

FIG. 3. Possible locations of present-day global liquid water layers in the icy moons of Jupiter are shown here as a function of depth: (1) completely frozen; (2) three-layered structures where the liquid layer cannot be in direct contact with the silicate floor; (3) thick upper icy layer $(>10 \mathrm{~km})$ and a deep ocean in contact with the rocky mantle; (4) very thin upper icy layer (3-4 km). Cases 3 and 4 are the most probable for Europa. Case 2 is expected for Ganymede and Callisto (from Blanc et al., 2009). Color images available online at www.liebertonline.com/ast

has been hypothesized on Enceladus and Europa. The Cassini spacecraft detected active plumes jetting from localized fractures at the south pole of Enceladus since the 2005 flybys (Dougherty et al., 2006). The composition of these plumes positively enhances the potential habitability of this moon because it includes $\mathrm{CO}_{2}, \mathrm{CO}, \mathrm{CH}_{4}, \mathrm{~N}_{2}$, and other volatiles containing chemical block elements. Two opposite models have been proposed to explain the water-ice geysers. Both are related to the tectonic activation of the icy crust, possibly found in the "tiger stripes," deep canyons located in the south polar region and viewed as conduits for the material forming the atmosphere. One of the models predicts the formation of the plumes by the dissociation of gas clathrates when the crust is fractured (Kieffer et al., 2006, Kieffer and Jakosky, 2008). Liquid water reservoirs are not necessary in this hypothesis, except if it is assumed that initially the clathrates are formed from gassy aqueous solutions. The other model requires a higher thermal gradient and the formation of a liquid ocean (Nimmo and Pappalardo, 2006; Porco et al., 2006). The latter model is supported by the discovery in the jets or in the E-ring of ammonia (Waite et al., 2009) and salts (Postberg et al., 2009), two compounds that depress the melting point of the aqueous system and destabilize the clathrate hydrates (Sloan and Koh, 2008).

Interaction between shallow aqueous reservoirs and the icy surfaces has been suggested in the chaos terrains of Europa as well (Schmidt et al., 2011). Shallow reservoirs have been suggested by comparing the chaos terrains with terrestrial ice shelves. The interpretation of the geological features that characterize Thera Macula, for instance, suggests that it is forming due to collapses of ice crust that occur when a liquid reservoir develops at shallow depths. These reservoirs are hypothesized to form by partial melting, when warm ice rises through the crust and causes it to thaw. Parts of the crust containing depressor impurities, such as sulfate salts, will be more prone to melting. The liquid lenses push the ice and produce cracks that disrupt the surface (Schmidt et al., 2011). The interaction between deep water with endogenous liquids may involve heat transfer, chemical exchange, or both. In the second case, materials from the environment in contact with the rock may have access to the surface through these features.

On Ganymede, the situation is quite different because there is no evidence of any present activity or even recent features that might suggest the existence of shallow reservoirs. No evidence for recent cryovolcanic resurfacing has been identified thus far. Only locally restricted scalloped depressions called paterae adjacent to Ganymede's bright terrain, which could represent caldera-like features (Lucchita, 1980; Schenk and Moore, 1995; Collins et al., 1998a; Kay and Head, 1999), are thought to be strong evidence for cryovolcanic features that appear in Ganymede's history. At least one of them was interpreted as an icy flow that produced smooth bright lanes within Ganymede's grooved terrain (Giese et al., 2001; Schenk et al., 2001). This suggests that cryovolcanism in combination with tectonic processes may have played some role in the past for the formation of the bright terrain (Collins et al., 1998a). It is not probable, however, that a heat pulse can reach the upper crust at present or that it could produce the kind of near-surface cryomagmatism as predicted on Europa. The formation of the resurfaced younger terrains, that is, the grooved terrain, has been aged to be on average $\sim 2$ billion years old (Zahnle et al., 2003) but may have formed somewhere between 1 and 3.6 billion years ago. Large uncertainties remain because of a factor of 3 uncertainty in the impactor flux (Zahnle et al., 2003; Patterson et al., 2010 and the references therein). Ganymede's grooved terrain was formed by tectonism during a global expansion episode of the moon, probably driven by tidal stress (Collins et al., 1998b; Pappalardo et al., 1998) and potentially due to the differentiation of Ganymede's interior. Since Ganymede is so large, a large volume of high-pressure ice phases in the interior displaced to the outside is expected to cause a significant volume change and thus cause isotropic tensile stress over the entire surface (Squyres, 1980; Mueller and McKinnon, 1988). During the emplacement of the grooved terrain, cryovolcanic extrusions may have been erased by tectonism or mass wasting in an early stage of the moon's evolution (Pappalardo et al., 1998).

As a conclusion, the geological evidence on Ganymede does not support the existence of shallow liquid reservoirs today. Still, such an occurrence cannot totally be ruled out because it must be kept in mind that most of the Galileo data 
was acquired at medium spatial resolution, impeding the detection of small features. Nonetheless, the very old age of the youngest surfaces (one hundred million years at leastsee below) is a very strong argument against present or recent activity such as the ones detected on Europa and Enceladus. Hence, we suggest that there is only a remote chance of having shallow reservoirs in the first few kilometers of the icy crust of Ganymede at present.

\section{Exchange Processes from Space to Habitable Zones}

When considering the transport processes that link the surrounding space environment, the surface of the moon, and the ocean, it is the thickness of the outer crust that is crucial. It is one of the most important factors that determine the timescale needed for a particle at the surface to penetrate to the ocean. Another major factor is the temperature profile within the ice shell because it affects the viscosity of the crust and, thus, the vigor of convective motions within the ice. To better constrain both the thickness and the exchange processes that may occur in the shell, it is necessary to obtain an estimate of the age of the crust.

The two major geological units on Ganymede-old, dark, densely cratered plains and young, bright, grooved plainsshow tectonic structures that imply episodes of past tectonism (e.g., Shoemaker et al., 1982; Pappalardo et al., 2004; Prockter et al., 2010; Stephan et al., 2013). However, the intense superimposed impact crater density, even in the bright terrains, implies that Ganymede's surface is mostly old and lacks any form of recent geological activity such as cryovolcanism or tectonism. Based on cratering records, it is agreed that the crust is at least a few hundreds of millions of years old in the youngest regions and at least $\sim 1$ billion years old in the oldest regions (see Zahnle et al., 2003). It is worth noting here that, for an age that exceeds $10^{8}$ years, the 2012 National Research Council Space Studies Board report (Committee on Planetary Protection Standards for Icy Bodies in the Outer Solar System, 2012) states that "the geologic timescale for delivery of surface materials into potentially habitable subsurface environments vastly exceeds the timescale of biological exploration." In the following sections, quantitative arguments will be given that strengthen this statement in the specific case of Ganymede.

\subsection{The thickness of the icy shell}

To our knowledge, the only constraint on the thickness of the crust that is based on direct measurements comes from the Galileo magnetic field data. These data suggest that the icy crust could be about $150 \mathrm{~km}$ thick (Kivelson et al., 2002). Other Galileo measurements and more recent observations of Ganymede did not allow the ice thickness to be directly constrained. In particular, the density contrast between liquid water and ice is too small to be resolved by Galileo gravity field data (Anderson et al., 1996). The youngest surface of Ganymede being older than 100 million years, the thickness of the icy layer must exceed $50 \mathrm{~km}$ [thickening scales roughly as $t^{1 / 2}$ (Kirk and Stevenson, 1987)]. In planetary mantles, diffusion is rapidly replaced by convection, which results in heat expellation from the deep interior. In the specific case of icy mantles, this transition from conduction to convection occurs once the icy mantle is a few tens of kilometers thick (Kirk and Stevenson, 1987; Grasset and
Sotin, 1996). In the case of Ganymede, all models agree on the fact that convection must occur, because the old age of the crust, which indicates a long period without major resurfacing, has permitted such a thickening.

In the case of convection, ice particles are moving, which forms upwelling and downwelling plumes. But the large temperature difference from top to bottom implies a huge viscosity contrast of several orders of magnitude (Durham et al., 2010). In that specific case, convection operates in the "stagnant lid regime" (Fig. 4). The convective zone is confined to a warm sublayer overlain by a stagnant layer that is purely diffusive (Spohn and Schubert, 2003). In Fig. 4, the different sublayers that exist in the stagnant lid regime are illustrated (not to scale).

Spohn and Schubert (2003) calculated models of ice and ocean thickness of Ganymede under the assumption of equilibrium between radiogenic heat production and surface heat flux. It is concluded that the ice-I layer on Ganymede is $60-80 \mathrm{~km}$ thick in the case of an $\mathrm{H}_{2} \mathrm{O}-\mathrm{NH}_{3}$ ocean. It is very hard to envisage a thinner icy layer, because ammonia is the most powerful antifreeze available in the Solar System (Grasset and Pargamin, 2005; Choukroun and Grasset, 2010). In the case of pure $\mathrm{H}_{2} \mathrm{O}$, the ice thickness would exceed the above values. Ocean thickness varies from $50 \mathrm{~km}$ at $130 \mathrm{~km}$ in depth in the case of pure water, up to $230 \mathrm{~km}$ at about $60 \mathrm{~km}$ depth if $\mathrm{NH}_{3}$ were added to the liquid. All other studies are in good agreement with these estimates (Pappalardo et al., 2004; Schubert et al., 2010 and references therein). Spohn and Schubert (2003) also suggested that $65-80 \%$ of the total ice shell is taken up by the stagnant lid (the actual number depending on the assumed ammonia content), which implies that at least the upper $30 \mathrm{~km}$ of Ganymede's ice shell is conductive even if the sublayer is convective.

Finally, further evidence of the large thickness of the icy layer comes from geological observations. In Ganymede's dark terrain, arcuate and radial furrows are ubiquitous tectonic features that were generated by large basin-forming impact events (Shoemaker et al., 1982; Pappalardo et al., 2004; Prockter et al., 2010; Stephan et al., 2013). Topographic analysis in which Galileo SSI stereo images were used showed that furrows have a maximum topographic height of $1.5 \mathrm{~km}$ and are up to $1 \mathrm{~km}$ deep (Giese et al., 1998). Shadow measurements revealed comparable values (Prockter et al., 2010 and references therein). At the time of formation of these furrows, possibly on the order of $\sim 4$ billion years ago (Neukum et al., 1998; Zahnle et al., 1998, 2003), Ganymede's lithosphere overlying a plastic asthenosphere was estimated to be $\sim 10 \mathrm{~km}$ thick but became thicker with time $(\sim 75$ $100 \mathrm{~km}$ ), as inferred from topography of the younger Gilgamesh basin (McKinnon and Melosh, 1980; Schenk et al., 2004).

\subsection{Exchange from space to the first micrometers}

Ganymede is "weathered" by trapped jovian charged particle populations of electrons and ions, and by solar UV photons. Radiolysis and photolysis, the chemical alteration of the surface by charged particles and solar UV photons, respectively, occur on the surfaces of the moons (see, e.g., the review by Johnson et al., 2004 and references therein). Radiolysis can alter the optical surface on short timescales and can produce chemical changes at micron to meter depths. Other processes at the surface (e.g., meteorite gardening, sublimation, and burial) can bury or trap chemically altered material 


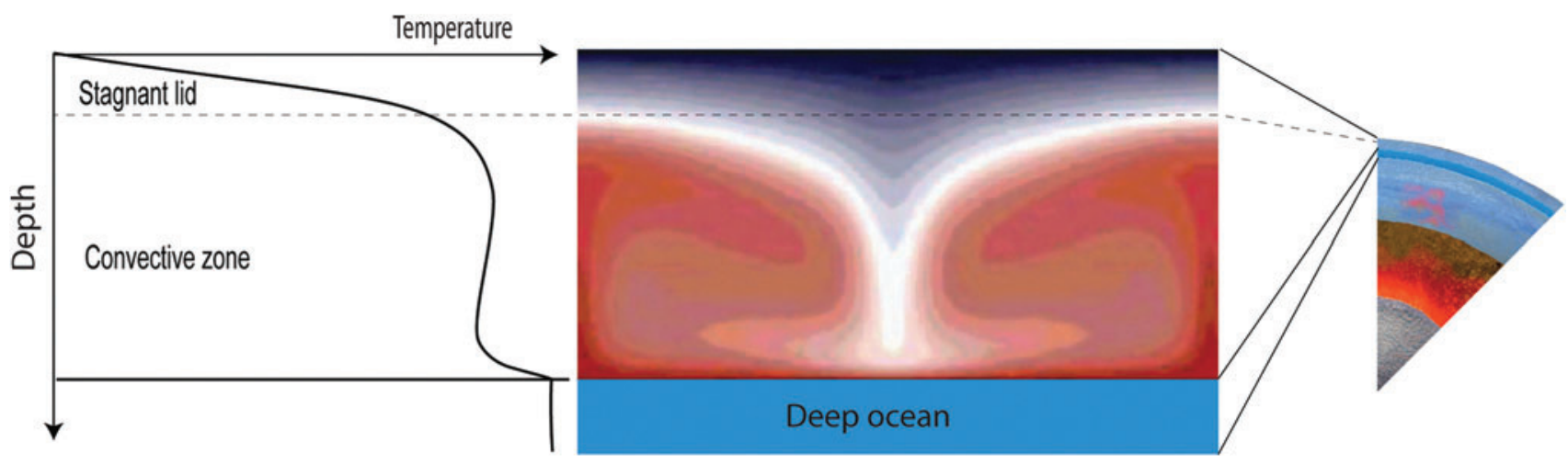

FIG. 4. Left: Averaged temperature profile from the surface to the ocean of an icy satellite (not to scale). The icy shell is split into an upper lid where most of the thermal (and viscosity) contrast occurs, and a convective sublayer. The convective sublayer is close to isothermal, except on top and bottom where two thermal boundary layers exist. Center: Convective patterns within an icy shell cooled from above and possessing strong viscosity contrasts. In this figure, the red colors stand for warmer temperatures and lower viscosity ice, and gray and white represent colder temperatures and stiffer ice. The convective sublayer is always located below a cold lid on top. Upwelling (in red) and downwelling (gray and white) through which a material can be transported efficiently are clearly visible. Right: Location of the crust on Ganymede. For the purpose of clarity, the convecting sublayer where the plumes are located is drawn here such that it occupies a major part of the icy shell. On Ganymede, it may be the opposite (see text for details). Color images available online at www.liebertonline.com/ast

at greater depths than the radiation penetration depths (see Cooper et al., 2001; Johnson et al., 2004). In addition, the solid surface is physically altered by impact processes that affect the shallow crust from meter to kilometer depths. Lanzerotti et al. (1978) undertook experiments to quantify the effect of energetic charged particle irradiation on low-temperature ices, such as on Ganymede. They discovered the process of "sputtering," where energetic particles bombard the surface, displace new particle species, and release energy.

In terms of the charged particle bombardment, there are hemispherical asymmetries as shown in the reflectance spectra of the satellites-for example, the leading versus trailing regions. In the jovian magnetosphere, the plasma is nearly corotating with the planet; hence the plasma "overtakes" the moon in its orbit. This produces a preferentially "trailing hemisphere" interaction between the satellite surface, with the ions and electrons trapped in the jovian radiation belts sweeping past the moon. To quantify the effect of particle radiation, we need to know the nature of the interaction between satellite and magnetosphere and what the characteristics of the particle environment are at a given moon.

For Ganymede, we know that there is an internally driven magnetic field and miniature magnetosphere. This magnetic field provides some modification of the particle flux toward a satellite's surface, allowing more direct access at the poles than at the equator. Ganymede is also observed to have latitudinal variations in reflectance with contrast between the equatorial and polar cap regions. This effect has been attributed to plasma bombardment (Johnson, 1985, 1997) and more recently suggested to be associated with the channeling of the jovian particles to the Ganymede surface predominantly on Ganymede's “open" (i.e., connected to Jupiter's) magnetic field lines near the poles (Johnson, 1997; Khurana et al., 2007). At Ganymede, the energetic particle access to the surface has been modeled in detail by Cooper et al. (2001), who found that, except in the magnetically shielded region near Ganymede's equator, the energetic electrons are the dominant carriers of energy for chemical radiolysis at the surface (with smaller relative inputs from protons and the heavy ions). In the equatorial regions, heavy ions have preferential access to the surface, but with much lower rates than in the open polar cap regions. Jovian energetic electrons above tens of kiloelectronvolts are completely excluded, while $>10 \mathrm{MeV}$ protons can penetrate but with much lower, in relative intensity, energy flux and sputtering rates.

From the charged particle environment knowledge and global bombardment pattern described above for Ganymede, the radiation dose as a function of depth at each location can be determined. The dose-depth relationship depends on species, energy, angle of incidence, and surface properties. The spatial bombardment distributions across the surface of the satellite depend both on the energy, mass, and charge of the particles and on the influence of the electric and magnetic fields near the moons. The "projected range" is the depth to which a given particle penetrates a given surface, and depends upon particle type and energy (or "stopping power" of the surface). Heavy ions have smaller penetration depth, and fast protons and electrons have the largest. For example, $10 \mathrm{keV} \mathrm{S}^{+}, \mathrm{H}^{+}$, and electrons penetrate ice to $\sim 0.03,0.3$, and $10 \mu \mathrm{m}$, respectively (Cooper et al., 2001). The dose (or deposited energy per unit volume) versus depth can then be obtained from the stopping power. The dose versus depth rate has been calculated for Ganymede (see Cooper et al., 2001). The computed energetic particle doses at depths up to a few microns are dominated by ions, and the electrons dominate up to tens of microns. Thermal ions that reach the surface only modify the immediate surface layer $(\sim 0.01$ micron). These vary spatially (leading vs. trailing hemispheres and polar vs. equatorial regions as discussed above). Figure 5a shows the time in years at each depth to accumulate a dose of $\sim 100 \mathrm{eV}$ per $16 \mathrm{amu}$ (mass of O). Regolith growth time is (relatively) much faster; hence the regolith grows much more quickly than radiolytic products accumulate. However, modeling work at Europa (Bierhaus et al., 2001; Moore et al., 2004) has suggested that the regolith growth time may be slower than the rates presented by Cooper et al. (2001); thus regolith production rates at Ganymede may be less than that suggested by Fig. 5. The icy 
surface can trap molecules, though, and may be a substantial reservoir of buried radiation-altered material.

No organism can survive on the surface of Ganymede over a long period of time because of the high-radiation environment. However, endolithic organisms may penetrate the subsurface of Ganymede to survive and may even be able to repair cell damage caused by radiation using slow metabolism (e.g., Nicholson et al., 2000; Bagwell et al., 2008). Nevertheless, even these organisms would have to bury deep into the subsurface on a timescale sufficiently short to avoid lethal cell damage due to the very low temperature and pressure and the high irradiation environment. The depth profiles of volume dosage rates (megarad per month) from the measured energetic particles (see Cooper et al., 2001 for more details) are shown in Fig. 5b. Europa is also shown for purposes of comparison. As Cooper et al. (2001) purported, certain organisms may survive for several days or more at Europa. Now, taking into account the fact that material might be partially covered by spacecraft parts, that the radiation inactivation is depending on the temperature (lower temperatures require higher dose), and radiation doses are lower on Ganymede, survival may conceivably be a few weeks or even months on Ganymede. However, such timescales will always be much shorter than the timescales that bury surface molecules to meter depths by (for example) meteoritic impacts.

\subsection{Exchange processes through the stagnant lid}

Underneath the regolith, the icy crust is composed of several layers that a downward-migrating organism would have to traverse. As said in 3.1, the upper part of the icy crust is made of a conductive layer, called the stagnant lid, below which the convection occurs (see Fig. 4). In this lid, cold ice is brittle, and no fluid motions are possible. Once a particle or a microorganism has been incorporated in the first micrometers, there are only two possible mechanisms to go through the lid: either by diffusion or through a tectonic feature breaking the entire lid if it were possible. The first mechanism is not realistic, especially over timescales relevant to microorganisms. Diffusive distances are always proportional to the square root of the product of chemical diffusivity and time. Thus, even when using a rather high value for the chemical diffusivity of ices (about $10^{-12} \mathrm{~m}^{2} / \mathrm{s}$ ), the time needed to go through $100 \mathrm{~m}$ of ice is about 300 million years. Following the discussion above, it would require about 28,000 billion years to go through the $30 \mathrm{~km}$ of the lid in the model of Spohn and Schubert.

Thus, the only way to go through the lid relies on the presence of geological activity. The main geological processes occurring on Ganymede are (a) impact cratering, (b) tectonism, and (c) cryovolcanism. Cryovolcanic activity is generally considered as a way to expel deep materials from the interior to the surface. However, in a few cases, such as when water-rich melts with density greater than ice are present, downward percolation through the upper shell and even down to the ocean could be possible (Pappalardo and Barr, 2004, in the case of Europa). On Ganymede, there are indeed a few indications that would suggest cryovolcanic activity in the past (Collins et al., 1998a; Pappalardo et al., 2004), but there are no observations of any present activity. To allow water-rich melts through the entire lid at present is not realistic, since there is no evidence of activity on the surface. For these reasons, this process has not been considered further in this work.
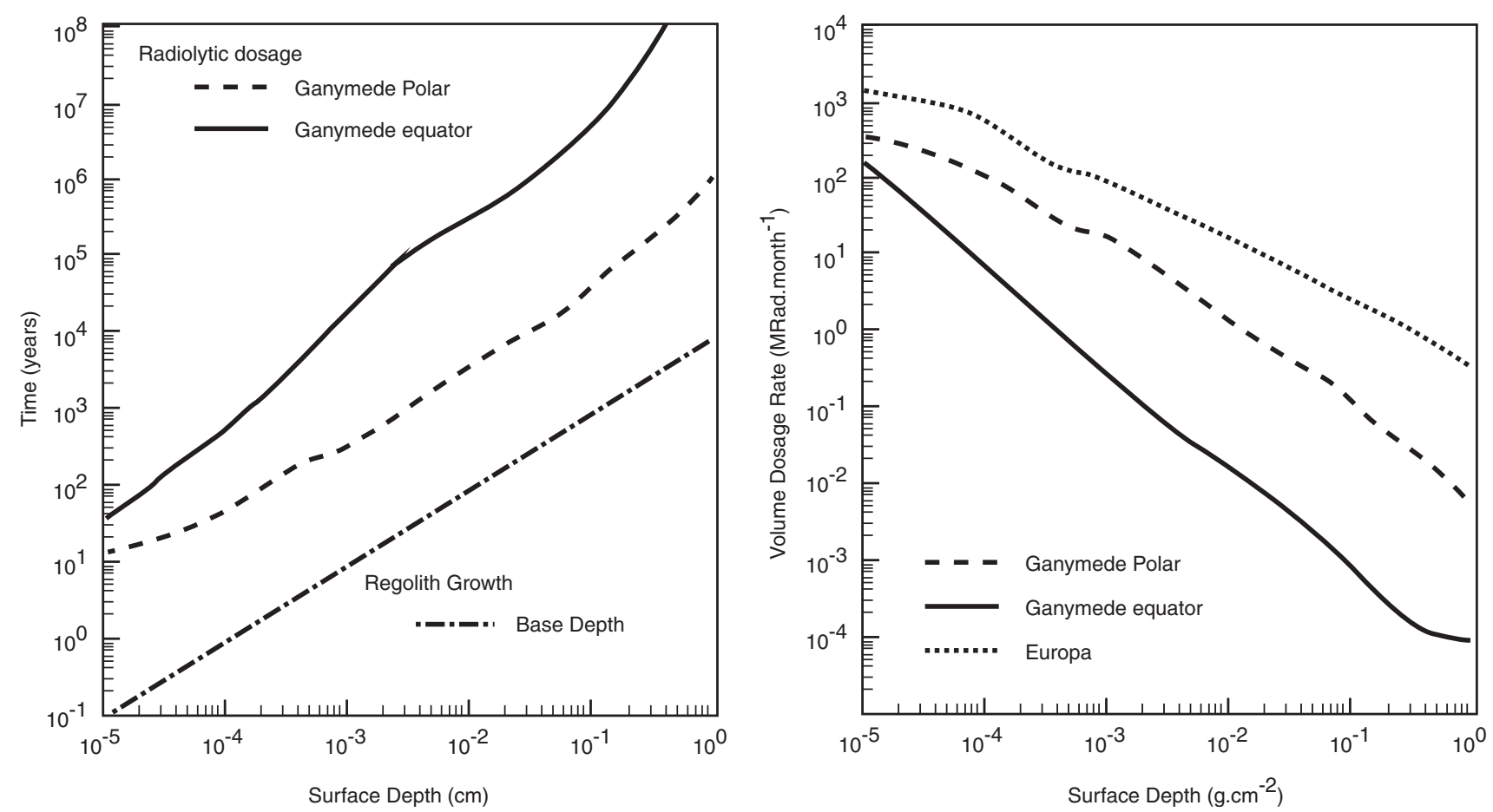

FIG. 5. Left: Time vs. depth for accumulating a significant dose, $100 \mathrm{eV}$ per $16 \mathrm{amu}$, compared to the time for the regolith to grow to that depth. Right: Depth profiles for total volume dosage rate in megarad (Mrad) per month for irradiation of pure $\mathrm{H}_{2} \mathrm{O}$ ice (source: Cooper et al., 2001). 
Impact as a candidate process to cross the stagnant lid can also be ruled out. During an impact, a transient cavity forms with a depth approximately one-third to one-quarter of the transient crater diameter (Melosh and Ivanov, 1999). Impacts creating craters with diameters of 10-20 km that have formed in substantial numbers over the past $\sim 3$ billion years (e.g., Zahnle et al., 2003) and that are common on both terrain types on Ganymede require kilometer-sized projectiles. These could only reach depths of a few kilometers, probably not enough to penetrate to the top of Ganymede's convective zone in the upper icy layer, which is assumed to be located at least a few tens of kilometers deep at present (see 3.1). The formation of impact basins like Gilgamesh, Ganymede's largest impact structure, which has an assumed nominal crater rim diameter of $\sim 600 \mathrm{~km}$ and a transient excavation depth of 150-200 km (Melosh and Ivanov, 1999; Schenk et al., 2004), is a rare event in the history of the evolution of the jovian system. Gilgamesh is on the order of $\sim 0.8-3.8$ billion years old, depending of the cratering chronology model applied (Neukum et al., 1998; Zahnle et al., 1998, 2003). It can be excluded therefore that the impact of a meter- or decameter-sized object could help to transfer materials from the surface to the convective zone below the lid.

Topography in grooves in the light terrain reaches a maximum of $\sim 700 \mathrm{~m}$. This estimate is based on stereo analysis and photoclinometry (Giese et al., 1998; Pappalardo et al., 2004 and references therein). Nimmo et al. (2002) used Galileo SSI stereo images to measure the elastic thickness in tectonized areas, which is an indicator of how deep tectonic structures can extend until the brittle-toductile layer is reached. The authors found a value of $\sim 1-$ $2 \mathrm{~km}$ at the time of groove formation (Nimmo et al., 2002 and references therein). Indeed, gravity and pressure of the overlying crust heal out fractures at that depth. The morphology of the post-groove Gilgamesh basin reveals, however, that Ganymede's brittle layer has thickened with time so that the brittle-ductile transition today is likely at greater depths than at the time when the bright grooved terrain formed (e.g., Schenk et al., 2004) but still very small compared to the probable thickness of the stagnant lid. Thus, the probability of dragging any material down to the convective sublayer through present tectonic features is negligible. It is worth mentioning that it may have been different in the past. Lee et al. (2005) showed that some fractures on Europa could penetrate down to a few tens of kilometers. The high strain rates that are implied are not consistent with the physical state of Ganymede at present but may have been applicable in the past.

\subsection{Exchange processes through the convecting sublayer}

In this section, an estimate of the time required for any material to go from the top of the convective sublayer (bottom of the stagnant lid) to the liquid layer below is proposed. Modeling of convection within the icy shell permits a prediction of the velocities within the convective zone. By using the simplest context of a pure ice mantle with constant viscosity, velocities are directly related to the vigor of convection when using the global Rayleigh number (Turcotte and Schubert, 2002). This assumption of constant viscosity is in fact close to reality, because the fluid in the convective sub- layer is almost isothermal [see Fig. 4 (left) or Fig. 2 in Spohn and Schubert (2003)]. More-complex modeling is possible and provides similar estimates. As an example, if one uses the Spohn and Schubert (2003) parameters for a convecting shell about $50 \mathrm{~km}$ thick (the lid above should be a few tens of kilometers), and a low viscosity of $10^{12} \mathrm{~Pa} \cdot \mathrm{s}\left(10^{11} \mathrm{~Pa} \cdot \mathrm{s}\right.$ is at the melting point), one obtains a Rayleigh number close to $10^{9}$ and a velocity close to $7 \mathrm{~m}$ /year (about 50 times more than on Earth). In this case, it would take about 7000 years to cross the convective layer along a cold descending plume. In fact, numbers vary from a few thousand years to about 100,000 years depending on the rheology adapted for the icy material.

This estimate of 7000 years must be considered as the shortest time required for exchanging material though the convective sublayer of Ganymede's icy mantle at present. A few meters per year is the highest velocity that can be envisaged. It is worth noting that it does not mean that the material descending along a cold plume goes directly to the ocean. Once the material reaches the bottom zone of the icy layer it moves horizontally in the lower thermal boundary layer until it rises again along a thermal upwelling. To descend into the ocean requires either diffusive processes through the thermal boundary layer or downward propagation through cracks. Diffusive processes are extremely slow and would take several thousands of years at least. However, fractures could potentially propagate from the iceocean interface into the ice shell (Crawford and Stevenson, 1988) all the way through the lower thermal boundary layer. Such an occurrence is uncertain, but it may provide an efficient way locally to propagate material from the convecting zone to the ocean.

As revealed by its surface morphology, Ganymede had periods in its evolution involving higher heat production rates than those at present. These stages of evolution could be related to differentiation processes and an associated heat pulse (Kirk and Stevenson, 1987; Mueller and McKinnon, 1988) or to enhanced tidal heating because of high orbital eccentricities, for example, due to the passage of resonances before formation of the Laplace resonance (Showman and Malhotra, 1997). However, as revealed by the old age of the surface even in younger terrains, the stages of enhanced internal heating that could facilitate the communication between surface and ocean occurred in the distant past. It is not impossible that enhanced internal heating could occur again in the future, but given the different factors involved such as the orbital states, it can only evolve on very long timescales (of the order of millions of years at least). Therefore, no changes in Ganymede's regime of tidal heating and heat transport mechanisms through the icy layer are expected at present or in Ganymede's near future.

\subsection{Summary}

In this paragraph, the different mechanisms and timescales of transport to the subsurface liquid water are summarized (Table 2). First, it is very difficult for a microorganism to penetrate through the regolith over a reasonable timescale, except in the case of an impactor (in the event the energy delivered after the impact does not destroy all microorganisms). Second, it has been shown that the upper icy layer must be at least $50 \mathrm{~km}$ thick; therefore it is implausible to 
Table 2. Processes of Downward Migration through the Sublayers of the Icy Shell above the Ocean

\begin{tabular}{|c|c|c|c|c|}
\hline $\begin{array}{l}\text { Layer (from top } \\
\text { to bottom) }\end{array}$ & $\begin{array}{l}\text { Minimal } \\
\text { thickness }\end{array}$ & $\begin{array}{c}\text { Downward migration } \\
\text { process }\end{array}$ & $\begin{array}{l}\text { Time duration } \\
\text { (years) }\end{array}$ & Comments \\
\hline Regolith & $\begin{array}{l}\text { First centimeters } \\
\text { of the crust }\end{array}$ & $\begin{array}{l}\text { Diffusion } \\
\text { Impact }\end{array}$ & $\begin{array}{l}\text { A few years } \\
<<1 \text { year }\end{array}$ & $\begin{array}{l}\text { Impact is the only way } \\
\text { to introduce } \\
\text { microorganisms into } \\
\text { the regolith in a short } \\
\text { timescale. }\end{array}$ \\
\hline $\begin{array}{l}\text { Icy crust (stagnant lid) } \\
\text { and upper thermal } \\
\text { boundary layer }\end{array}$ & $\begin{array}{l}\text { From a few } \\
\text { kilometers } \\
\text { to a few tens } \\
\text { of kilometers }\end{array}$ & $\begin{array}{l}\text { Diffusion } \\
\text { Tectonism }\end{array}$ & $\begin{array}{l}\text { Several millions } \\
\text { of years } \\
<<1 \text { year but } \\
\text { extremely } \\
\text { unlikely through } \\
\text { the entire lid }\end{array}$ & $\begin{array}{l}\text { No way envisaged to go } \\
\text { through the lid in a } \\
\text { short timescale. } \\
\text { Geological evidence } \\
\text { demonstrates that the } \\
\text { faults cannot propagate } \\
\text { through the total } \\
\text { thickness of the crust } \\
\text { because it is too thick. }\end{array}$ \\
\hline Convecting layer & $\begin{array}{l}\text { From a few } \\
\text { kilometers } \\
\text { to a few tens } \\
\text { of kilometers }\end{array}$ & $\begin{array}{l}\text { Convective transport up } \\
\text { to a few meters per } \\
\text { year along a descending } \\
\text { cold plume }\end{array}$ & $\begin{array}{l}\text { A few thousand } \\
\text { years }\end{array}$ & $\begin{array}{l}\text { Duration estimate is based } \\
\text { on a very conservative } \\
\text { approach regarding the } \\
\text { vigor of convection. }\end{array}$ \\
\hline $\begin{array}{l}\text { Lower thermal } \\
\text { boundary layer }\end{array}$ & $\begin{array}{l}\text { A few hundred } \\
\text { meters }\end{array}$ & $\begin{array}{l}\text { Almost } \\
\text { impossible-diffusion } \\
\text { could be envisaged } \\
\text { Fractures }\end{array}$ & $\begin{array}{l}\text { A few thousand years } \\
\text { at least if diffusion } \\
\text { is feasible } \\
<<1 \text { year }\end{array}$ & $\begin{array}{l}\text { Upward thermal buoyancy } \\
\text { impedes downward } \\
\text { migration. } \\
\text { No evidence that such } \\
\text { fractures exist. }\end{array}$ \\
\hline
\end{tabular}

have direct contact between the surface of the moon and the liquid water reservoirs, because tectonic features cannot go deeper than a few kilometers. It is also clear that the transition from brittle to ductile domains is shallower than the transition from the stagnant lid to the convecting sublayer, which indicates that there is no way to reach the convecting sublayer over timescales smaller than millions of years. Even if this could occur, it would still require a few thousands of years for any material to be dragged down along cold plumes in the convecting sublayers. Finally, it is not certain that exchange processes are possible in the lower thermal boundary layer. The only possible process would be diffusion, indicating that a few thousands of years must still added to the timescale. Based on these different estimates, a total of a few millions of years is required to exchange material from the surface of the moon to the liquid layer because of the thickness of the upper lid.

\section{Conclusion}

In this paper, we have analyzed the various evidence gathered by previous measurements at the giant icy moon Ganymede that are related to exchange processes within the upper layers of the surface. We have given a detailed overview of its characteristics and probability of occurrence of liquid water reservoirs within the moon. It has been previously shown that the deep liquid ocean must be present but very deep, while shallow liquid reservoirs within the crust are not expected because of the old age of the crust. Conservative estimates of the time duration for each possible mechanism of downward migration through the icy layer have also been quantified to place constraints on the planetary protection measures for this moon.
Ganymede is of strong interest regarding organics and its potential for hosting a habitable ocean. It displays no evidence at the surface of recent activity that could propagate biological contamination beyond a spacecraft landing zone, in spite of the evidence of past and possibly present endogenic activity. Nonetheless, the different constraints arising from Galileo data and the structure and dynamics modeling of the upper layers that have been reviewed in this paper demonstrate that our Solar System's largest moon should not be considered as having a significant chance of being contaminated by a space mission. Following COSPAR's categorization, we suggest that Ganymede is definitely of significant interest relative to the process of chemical evolution and the origin of life, and there is only a remote chance that contamination by a spacecraft could compromise future investigations.

\section{Acknowledgments}

The authors would like to gratefully acknowledge the contribution of our colleagues K. Stephan and R. Wagner from DLR, Berlin.

\section{Abbreviation}

JUICE, JUpiter ICy moons Explorer.

\section{References}

Anderson, J.D., Lau, E.L., Sjogren, W.L., Schubert, G., and Moore, W.B. (1996) Gravitational constraints on the internal structure of Ganymede. Nature 384:541-543.

Bagwell, C.E., Milliken, C.E., Ghoshroy, S., and Blom, D.A. (2008) Intracellular copper accumulation enhances the growth 
of Kineococcus radiotolerans during chronic irradiation. Appl Environ Microbiol 174:1376-1384.

Barr, A.C. and Stillman, D.E. (2011) Strain history of the ice shells of the Galilean satellites from radar detection of crystal orientation fabric. Geophys Res Lett 214:773-778.

Bierhaus, E.B., Chapman, C.R., Merline, W.J., Brooks, S.M., and Asphaug, E. (2001) Pwyll secondaries and other small craters on Europa. Icarus 153:264-276.

Blanc, M., Alibert, Y., André, N., Atreya, S., Beebe, R., Benz, W., Bolton, S.J., Coradini, A., Coustenis, A., Dehant, V., Dougherty, M., Drossart, P., Fujimoto, M., Grasset, O., Gurvits, L., Hartogh, P., Hussmann, H., Kasaba, Y., Kivelson, M., Khurana, K., Krupp, N., Louarn, P., Lunine, J., McGrath, M., Mimoun, D., Mousis, O., Oberst, J., Okada, T., Pappalardo, R., Prieto-Ballesteros, O., Prieur, D., Regnier, P., Roos-Serote, M., Sasaki, S., Schubert, G., Sotin, C., Spilker, T., Takahashi, Y., Takashima, T., Tosi, F., Turrini, D., Van Hoolst, T., and Zelenyi, L. (2009) LAPLACE: a mission to Europa and the Jupiter System for ESA's Cosmic Vision Programme. Experimental Astronomy 23:849-892.

Bridgman, P.W. (1912) Water in the liquid and five solid forms under pressure. Proceedings of the American Academy of Arts and Sciences 47:441-558.

Bridgman, P.W. (1937) The phase diagram of water to $45,000 \mathrm{~kg} /$ $\mathrm{cm}^{2}$. J Chem Phys 5:964-966.

Brooks, N.J., Ces, O., Templer, R.H., and Seddon, J.M. (2011) Pressure effects on lipid membrane structure and dynamics. Chem Phys Lipids 164:89-98.

Choukroun, M. and Grasset, O. (2010) Thermodynamic data and modeling of the water and ammonia-water phase diagrams for planetary geophysics. J Chem Phys 133, doi:10.1063/1.3487520.

Citroni, M., Ceppatelli, M., and Bini, R. (2005) High pressure reactivity of propene. J Chem Phys 123, doi:10.1063/1.2109947.

Clark, K., Boldt, J., Greeley, R., Hand, K., Jun, I., Lock, R., Pappalardo, R.T., Van Houten, T., and Yan, T. (2011) Return to Europa: overview of the Jupiter Europa orbiter mission. Adv Space Res 48:629-650.

Collins, G.C., Head, J.W., and Pappalardo, R.T. (1998a) The role of extensional instability in creating Ganymede grooved terrain: insights from Galileo high-resolution stereo imaging. Geophys Res Lett 25:233-236.

Collins, G.C., Head, J.W., and Pappalardo, R.T. (1998b) Formation of Ganymede grooved terrain by sequential extensional episodes: implications of Galileo observations for regional stratigraphy. Icarus 135:345-359.

Committee on Planetary Protection Standards for Icy Bodies in the Outer Solar System. (2012) Assessment of Planetary Protection Requirements for Spacecraft Missions to Icy Solar System Bodies, National Academies Press, Washington, DC.

Cooper, J.F., Johnson, R.E., Mauk, B.H., Garrett, H.B., and Gehrels, N. (2001) Energetic ion and electron irradiation of the icy Galilean satellites. Icarus 149:133-159.

Coustenis, A. and Taylor, F.W. (2008) Titan: Exploring an Earthlike World, $2^{\text {nd }}$ ed., Series on Atmospheric, Oceanic and Planetary Physics Vol. 4, World Scientific Publishing, Singapore.

Crawford, G.D. and Stevenson, D.J. (1988) Gas driven water volcanism and the resurfacing of Europa. Icarus 73:66-79.

Dougherty, M.K., Khurana, K.K., Neubauer, F.M., Russell, C.T., Saur, J., Leisner, J.S., and Burton, M.E. (2006) Identification of a dynamic atmosphere at Enceladus with the Cassini magnetometer. Science 311:1406-1411.

Durham, W.B., Prieto-Ballesteros, O., Goldsby, D.L., and Kargel, J.S. (2010) Rheological and thermal properties of icy materials. Space Sci Rev 153:273-298.
Giese, B., Oberst, J., Roatsch, T., Neukum, G., Head, J.W., and Pappalardo, R.T. (1998) The local topography of Uruk Sulcus and Galileo Regio obtained from stereo images. Icarus 135:303-316.

Giese, B., Wagner, R., Neukum G., Pappalardo, R.T., Head, J.W., and the Galileo SSI team. (2001) The topography of bright/ dark terrain on Ganymede [abstract 1751]. In $32^{\text {nd }}$ Lunar and Planetary Science Conference, Lunar and Planetary Institute, Houston.

Grasset, O. and Pargamin, J. (2005) The ammonia-water system at high pressures: implications for the methane of Titan. Planet Space Sci 53:371-384.

Grasset, O. and Sotin, C. (1996) The cooling rate of a liquid shell in Titan's interior. Icarus 123:101-112.

Grasset, O., Dougherty, M.K., Coustenis, A., Bunce, E.J., Erd, C., Titov, D., Blanc, M., Coates, A., Drossart, P., Fletcher, L.N., Hussmann, H., Jaumann, R., Krupp, N., Lebreton, J.-P., PrietoBallesteros, O., Tortora, P., Tosi, F., and Van Hoolst, T. (2013) JUpiter ICy moons Explorer (JUICE): an ESA mission to orbit Ganymede and to characterise the Jupiter system. Planet Space Sci 78:1-21.

Gruner, S.M. (1989) Stability of lyotropic phases with curved interfaces. J Phys Condens Matter 93:7562-7570.

Hamman, S.D. (1982) The influence of pressure on ionization equilibria in aqueous solutions. J Solution Chem 11:63-68.

Hazen, R.M., Boctor, N., Brandes, J.A., Cody, G.D., Hemley, R.J., Sharma, A., and Yoder, H.S., Jr. (2002) High pressure and the origin of life. J Phys Condens Matter 14:11489-11494.

Hussmann, H., Sohl, F., and Spohn, T. (2006) Subsurface oceans and deep interiors of medium-sized outer planet satellites and large trans-neptunian objects. Icarus 185:258-273.

Iess, L., Jacobson, R.A., Ducci, M., Stevenson, D.J., Lunine, J.I., Armstrong, J.W., Asmar, S.W., Racioppa, P., Rappaport, N.J., and Tortora, P. (2012) The tides of Titan. Science 337:457-459.

Johnson, R.E. (1985) Polar frost formation on Ganymede. Icarus 62:344-347.

Johnson, R.E. (1997) Polar "caps" on Ganymede and Io revisited. Icarus 128:469-471.

Johnson, R.E., Carlson, R.W., Cooper, J.F., Paranicas, C., Moore, M.H., and Wong, M.C. (2004) Radiation effects on the surfaces of the Galilean satellites. In Jupiter: The Planet, Satellites and Magnetosphere, edited by F. Bagenal, T.E. Dowling, and W.B. McKinnon, Cambridge University Press, Cambridge, UK, pp 485-512.

Kargel, J.S., Kaye, J.Z., Head, J.W., Marion, G.M., Sassen, R., Crowley, J.K., Ballesteros, O.P., Grant, S.A., and Hogenboom, D.L. (2000) Europa's crust and ocean: origin, composition, and the prospects for life. Icarus 148:226-265.

Kay, J.E. and Head, J.W., III. (1999) Geologic mapping of the Ganymede G8 Calderas region: evidence for cryovolcanism [abstract 1103]. In 30 ${ }^{\text {th }}$ Lunar and Planetary Science Conference, Lunar and Planetary Institute, Houston.

Khurana, K.K., Papparlardo, R.T., Murphy, N., and Denk, T. (2007) The origin of Ganymede's polar caps. Icarus 191:193-202.

Kieffer, S.W. and Jakosky, B.M. (2008) Enceladus-oasis or ice ball? Science 320:1432-1433.

Kieffer, S.W., Lu, X.L., Bethke, C.M., Spencer, J.R., Marshak, S., and Navrotsky, A. (2006) A clathrate reservoir hypothesis for Enceladus' south polar plume. Science 314:1764-1766.

Kirk, R.L. and Stevenson, D.J. (1987) Thermal evolution of a differentiated Ganymede and implications for surface features. Icarus 69:91-134.

Kivelson, M.G. (2004) Moon-magnetosphere interactions: a tutorial. Adv Space Res 33:2061-2077. 
Kivelson, M.G., Khurana, K.K., Russell, C.T., Volwerk, M., Walker, R.J., and Zimmer, C. (2000) Galileo magnetometer measurements: a stronger case for a subsurface ocean at Europa. Science 289:1340-1343.

Kivelson, M.G., Khurana, K.K., and Volwerk, M. (2002) The permanent and inductive magnetic moments of Ganymede. Icarus 157:507-522.

Lammer, H., Bredehoft, J.-H., Coustenis, A., Khodachenko, M.L., Kaltenegger, L., Grasset, O., Prieur, D., Raulin, F., Ehrenfreud, P., Yamauchi, M., Wahlund, J.-E., Griesmaier, J.M., Stangl, G., Cockell, C.S., Kulikov, Y., Grenfell, J.-L., and Rauer, H. (2009) What makes a planet habitable. Astronomy and Astrophysics Review 17:181-249.

Lanzerotti, L.J., Brown, W.L., Poate, J.M., and Augustyniak, W.M. (1978) On the contribution of water products from Galilean satellites to the jovian magnetosphere. Geophys Res Lett 5:155-158.

Lee, S., Pappalardo, R.T., and Makris, N.C. (2005) Mechanics of tidally driven fractures in Europa's ice shell. Icarus 177: 367-379.

Lonsdale, P. (1977) Clustering of suspension-feeding macrobenthos near abyssal hydrothermal vents at oceanic spreading centers. Deep Sea Research 24:857-858.

Lucchita, B.K. (1980) Grooved terrain on Ganymede. Icarus 44:481-501.

Margosch, D., Ehrmann, M.A., Buckow, R., Heinz, V., Vogel, R.F., and Gänzle, M.G. (2006) High-pressure-mediated survival of Clostridium botulinum and Bacillus amyloliquefaciens endospores at high temperature. Appl Environ Microbiol 72:3476-3481.

McCord, T.B., Hansen, G.B., and Hibbitts, C.A. (2001) Hydrated salt minerals on Ganymede's surface: evidence of an ocean below. Science 292:1523-1525.

McKinnon, W.B. and Melosh, H.J. (1980) Evolution of planetary lithospheres: evidence from multiringed structures on Ganymede and Callisto. Icarus 44:454-471.

Melosh, H.J. and Ivanov, B.A. (1999) Impact crater collapse. Annu Rev Earth Planet Sci 27:385-415.

Michels, P.C. and Clark, D.S. (1997) Pressure-enhanced activity and stability of a hyperthermophilic protease from a deep-sea methanogen. Appl Environ Microbiol 63:3985-3991.

Moore, J.M., Schenk, P.M., Bruesch, L.S., Asphaug, E., and McKinnon, W.B. (2004) Large impact features on middle-sized icy satellites. Icarus 171:421-443.

Mueller, S. and McKinnon, W.B. (1988) Three-layered models of Ganymede and Callisto: compositions, structures and aspects of evolution. Icarus 76:437-464.

Neukum, G., Wagner, R., Wolf, U., Ivanov, B.A., Head, J.W., Pappalardo, R.T., Klemaszewski, J.E., Greeley, R., Belton, M.J.S., and the Galileo SSI team. (1998) Cratering chronology in the jovian system and derivation of absolute ages [abstract 1742]. In $29^{\text {th }}$ Lunar and Planetary Science Conference, Lunar and Planetary Institute, Houston.

Nicholson, W.L., Munakata, N., Horneck, G., Melosh, H.J., and Setlow, P. (2000) Resistance of Bacillus endospores to extreme terrestrial and extraterrestrial environments. Microbiol Mol Biol Rev 64:548-572.

Nimmo, F. and Pappalardo, R.T. (2006) Diapir-induced reorientation of Saturn's moon Enceladus. Nature 441: 614-616.

Nimmo, F., Pappalardo, R.T., and Giese, B. (2002) Effective elastic thickness and heat flux estimates on Ganymede. Geophys Res Lett 29, doi:10.1029/2001GL013976.
Pappalardo, R.T. and Barr, A. (2004) The origin of domes on Europa: the role of thermally induced compositional diapirism. Geophys Res Lett 31, doi:10.1029/2003GL019202.

Pappalardo, R.T., Head, J.W., Collins, G.C., Kirk, R.L., Neukum, G., Oberst, J., Giese, B., Greeley, R., Chapman, C.R., Helfenstein, P., Moore, J.M., McEwen, A., Tufts, B.R., Senske, D.A., Breneman, H.H., and Klaasen, K. (1998) Grooved terrain on Ganymede: first results from Galileo high-resolution imaging. Icarus 135:276-302.

Pappalardo, R.T., Collins, G.C., Head, J.W., Helfenstein, P., McCord, T.B., Moore, J.M., Prockter, L.M., Schenk, P.M., and Spencer, J.R. (2004) Geology of Ganymede. In Jupiter: The Planet, Satellites and Magnetosphere, edited by F. Bagenal, T.E. Dowling, and W.B. McKinnon, Cambridge University Press, Cambridge, UK, pp 363-396.

Patterson, G.W., Collins, G.C., Head, J.W., Pappalardo, R.T., Prockter, L.M., Lucchita, B.K., and Kay, J.P. (2010) Global geological mapping of Ganymede. Icarus 207:845-867.

Porco, C.C., Helfenstein, P., Thomas, P.C., Ingersoll, A.P., Wisdom, J., West, R., Neukum, G., Denk, T., Wagner, R., Roatsch, T., Kieffer, S., Turtle, E., McEwen, A., Johnson, T.V., Rathbun, J., Veverka, J., Wilson, D., Perry, J., Spitale, J., Brahic, A., Burns, J.A., DelGenio, A.D., Dones, L., Murray, C.D., and Squyres, S. (2006) Cassini observes the active south pole of Enceladus. Science 311:1393-1401.

Postberg, F., Kempf, S., Schmidt, J., Brilliantov, N., Beinsen, A., Abel, B., Buck, U., and Srama, R. (2009) Sodium salts in E-ring ice grains from an ocean below the surface of Enceladus. Nature 459:1098-1101.

Prockter, L.M., Lopes, R.M.C., Giese, B., Jaumann, R., Lorenz, R.D., Pappalardo, R.T., Patterson, G.W., Thomas, P.C., Turtle, E.P., and Wagner, R.J. (2010) Characteristics of icy surfaces. Space Sci Rev 153:63-111.

Rummel, J.D., Ehrenfreund, P., and Peter, N., editors. (2009) COSPAR Workshop on Planetary Protection for Outer Planet Satellites and Small Solar System Bodies, Committee On Space Research, Paris.

Sagan, C. and Coleman, S. (1966) Decontamination standards for martian exploration programs. In Biology and the Exploration of Mars, Space Science Board, National Academy of Sciences, Washington, DC, pp 470-481.

San Martín, M.F., Barbosa-Cánovas, G.V., and Swanson, B.G. (2002) Food processing by high hydrostatic pressure. Crit Rev Food Sci Nutr 42:627-645.

Schenk, P.M. and Moore, J.M. (1995) Volcanic constructs on Ganymede and Enceladus: topographic evidence from stereo images and photoclinometry. J Geophys Res 100:19009-19022.

Schenk, P.M., McKinnon, W.B., Gwynn, D., and Moore, J.M. (2001) Flooding of Ganymede's bright terrains by lowviscosity water-ice lavas. Nature 410:57-60.

Schenk, P.M., Chapman, C.R., Zahnle, K., and Moore, J.M. (2004) Ages and interiors: the cratering record of the Galilean satellites. In Jupiter: The Planet, Satellites and Magnetosphere, edited by F. Bagenal, T.E. Dowling, and W.B. McKinnon, Cambridge University Press, Cambridge, UK, pp 427-456.

Schmidt, B.E., Blankenship, D.D., Patterson, G.W., and Schenk, P.M. (2011) Active formation of 'chaos terrain' over shallow subsurface water on Europa. Nature 479:502-505.

Schubert, G., Anderson, J.D., Spohn, T., and McKinnon, W.B. (2004) Interior composition, structure and dynamics of the Galilean satellites. In Jupiter: The Planet, Satellites and Magnetosphere, edited by F. Bagenal, T.E. Dowling, and W.B. McKinnon, Cambridge University Press, Cambridge, UK, pp 281-306. 
Schubert, G., Hussmann, H., Lainey, V., Matson, D.L., McKinnon, W.B., Sohl, F., Sotin, C., Tobie, G., Turrini, D., and Van Hoolst, T. (2010) Evolution of icy satellites. Space Sci Rev 153:447-484.

Shoemaker, E.M., Lucchita, B.K., Wilhelms, D.E., Plescia, J.B., and Squyres S.W. (1982) The geology of Ganymede. In Satellites of Jupiter, edited by D. Morrison, University of Arizona Press, Tucson, pp 435-520.

Showman, A.P. and Malhotra, R. (1997) Tidal evolution into the Laplace resonance and the resurfacing of Ganymede. Icarus 127:93-111.

Sloan, E.D. and Koh, C.A. (2008) Clathrate Hydrates of Natural Gases, $3^{\text {rd }}$ ed., CRC Press, Boca Raton, FL.

Sohl, F., Spohn, T., Breuer, D., and Nagel, K. (2002) Implications from Galileo observations on the interior structure and chemistry of the Galilean satellites. Icarus 157:104-119.

Sohl, F., Choukroun, M., Kargel, J., Kimura, J., Pappalardo, R., Vance, S., and Zolotov, M. (2010) Subsurface water oceans on icy satellites: chemical composition and exchange processes. Space Sci Rev 153:485-510.

Spohn, T. and Schubert, G. (2003) Oceans in the icy Galilean satellites of Jupiter? Icarus 161:456-467.

Squyres, S.W. (1980) Volume changes in Ganymede and Callisto and the origin of grooved terrain. Geophys Res Lett 7:593-596.

Stephan, K., Jaumann, R., and Wagner, R. (2013) Geology of icy bodies. In The Science of Solar System Ices, Astrophysics and Space Science Library Vol. 356, edited by M.S. Gudipati and J. Castillo-Rogez, Springer, New York, pp 279-367.

Stern, S.A. and McKinnon, W.B. (2000) Triton's surface age and impactor population revisited in light of Kuiper Belt fluxes: evidences for small Kuiper Belt objects and recent geological activity. Astron J 119:945-952.

Stofan, E.R., Elachi, C., Lunine, J.I., Lorenz, R.D., Stiles, B., Mitchell, K.L., Ostro, S., Soderblom, L., Wood, C., Zebker, H., Wall, S., Janssen, M., Kirk, R., Lopes, R., Paganelli, F., Radebaugh, J., Wye, L., Anderson, Y., Allison, M., Boehmer, R., Callahan, P., Encrenaz, P., Flamini, E., Francescetti, G., Gim, Y., Hamilton, G., Hensley, S., Johnson, W.T.K., Kelleher, K., Muhleman, D., Paillou, P., Picardi, G., Posa, F., Roth, L., Seu, R., Shaffer, S., Vetrella, S., and West, R. (2007) The lakes of Titan. Nature 445:61-64.

Task Group on the Forward Contamination of Europa. (2000) Preventing the Forward Contamination of Europa, National Academies Press, Washington, DC.

Turcotte, D.L. and Schubert, G. (2002) Geodynamics, $2^{\text {nd }}$ ed., Cambridge University Press, Cambridge, UK.

Turtle, E.P., Del Genio, A.D., Barbara, J.M. Perry, J.E., Schaller, E.L., McEwen, A.S., West, R.A., and Ray, T.L. (2011) Seasonal changes in Titan's meteorology. Geophys Res Lett 38, doi:10.1029/2010GL046266.

Vuitton, V., Lavvas, P., Yelle, R.V., Galand, M., Wellbrock, A., Lewis, G.R., Coates, A.J., and Wahlund, J.-E. (2009) Negative ion chemistry in Titan's upper atmosphere. Planet Space Sci 57:1558-1572.

Wackett, L.P., Dodge, A.G., and Ellis, L.B.M. (2004) Microbial genomics and the periodic table. Appl Environ Microbiol 70:647-655.

Waite, J.H., Combi, M.R., Ip, W.-H., Cravens, T.E., McNutt, R.L., Kasprzak, W., Yelle, R., Luhmann, J., Niemann, H., Gell, D., Magee, B., Fletcher, G., Lunine, J., and Tseng, W.-L. (2006) Cassini Ion and Neutral Mass Spectrometer: Enceladus plume composition and structure. Science 311:1419-1422.

Waite, J.H., Lewis, W.S., Magee, B.A., Lunine, J.I., McKinnon, W.B., Glein, C.R., Mousis, O., Young, D.T., Brockwell, T., Westlake, J., Nguyen, M.J., Teolis, B.D., Niemann, H.B., McNutt, R.L., Perry, M., and Ip, W.H. (2009) Liquid water on Enceladus from observations of ammonia and ${ }^{40} \mathrm{Ar}$ in the plume. Nature 460, doi: 10.1038/nature08352.

Waite, J.H., Young, D.T., Westlake, J.H., Lunine, J.I., McKay, C.P., and Lewis W.S. (2010) High altitude production of Titan's aerosols. In Titan from Cassini-Huygens, edited by R.H. Brown, J.-P Lebreton, and J.H. Waite, Springer, Dordrecht, pp 201-214.

Zahnle, K., Dones, L., and Levison, H.F. (1998) Cratering rates on the Galilean satellites. Icarus 136:202-222.

Zahnle, K., Schenk, P., Levison, H., and Dones, L. (2003) Cratering rates in the outer Solar System. Icarus 163: 263-289.

Zimmer, C., Khurana, K.K., and Kivelson, M.G. (2000) Subsurface oceans on Europa and Callisto: constraints from Galileo magnetometer observations. Icarus 147:329-347.

Address correspondence to: O. Grasset Planetology and Geodynamics University of Nantes Houssiniere Street 2 Nantes 44322

France

E-mail: olivier.grasset@univ-nantes.fr

Submitted 9 April 2013 Accepted 7 September 2013 